\title{
Recomendaciones para la prevención de infecciones relacionadas con demoliciones, construcciones y remodelaciones
}

\author{
(Recommendations for prevention of infection related to demolition, \\ construction and renovation)
}

\author{
Fernando Moreno ${ }^{1 *}$, Ankie Schade ${ }^{1}$, Leonor Márquez¹, \\ Pamela Rivero ${ }^{1}$, Cristian Smith'. \\ 1Unidad de Prevención y Control de Infecciones Asociadas a la Atención de Salud. \\ Hospital Carlos van Buren de Valparaíso \\ *Auor para correspondencia:infeccionesintra.hcvb@redsalud.gov.cl \\ RECIBID0:15 de Enero de 2015 \\ APROBAD0:17 de Mayo de 2015
}

LOS AUTORES DECLARAN NO TENER CONFLICTO DE INTERESES

Palabras clave: infecciones, aspergilosis, construcción

Key words: infections, aspergillosis, construction

\section{RESUMEN}

Las demoliciones, construcciones $\mathrm{y} / 0$ remodelaciones pueden provocar infecciones intrahospitalarias en pacientes con factores de riesgos, especialmente aspergilosis pulmonar. En la presente guia se describen algunas medidas que pueden ayudar a mitigar estas infecciones en los distintos recintos hospitalarios

\section{INTRODUCCION}

Las demoliciones, construcciones y/o remodelaciones en el hospital son una situación de riesgo para los pacientes debido a que el polvo que generan puede ser el vehículo para microorganismos oportunistas $(1,2)$.

El grupo de mayor riesgo son los pacientes inmunocomprometidos y aquellos que tienen procedimientos invasores, como por ejemplo ventilación mecánica. Las infecciones más frecuentes son las producidas por hongos filamentosos, en especial Aspergillus, sin embargo también se reportan casos

\section{ABSTRACT}

Demolitions, constructions and renovations can produce nosocomial infections in patients with risk factors, specially lung aspergillosis. The following guide describes some actions that could help in the mitigation of these infections in the different hospitals.

de infecciones por Legionella pneumophila y micobacterias atípicas $(1,3,4,5)$.

Es por lo anterior que estos proyectos suponen un reto muy especial y requieren para la seguridad de los pacientes de la participación de un grupo multidisciplinario, el cual debe incluir a los integrantes de la Unidad de prevención y control de IAAS, quienes deberán participar en todas las fases de las obras, asegurando el cumplimiento adecuado de las medidas de mitigación.

El objetivo de esta guía es proporcionar un documento que establezca las medidas de prevención 
Recomendaciones para la prevención de infecciones relacionadas con demoliciones, - F. Moreno et al

para diminuir el riesgo de infecciones relacionadas a remodelaciones, construcciones y/0 demoliciones, en particular las fúngicas invasoras en pacientes inmunocomprometidos o con factores de riesgos para estas infecciones.

\section{RECOMENDACIONES GENERALES $(6,7,8)$}

- La unidad de prevención y control de IAAS, en conjunto con el centro de responsabilidad, más el departamento de operaciones deben autorizar el inicio de la construcción previa inspección de las medidas de mitigación instauradas.

- La unidad de prevención y control IAAS categorizará el riesgo y recomendará las medidas especificas (Tabla 1).

- Si el riesgo es alto, los pacientes se podrán trasladar a otro servicio u hospital.

- Toda obra debe quedar aislada de las áreas clínicas y de la circulación del público con el fin de asegurar la no contaminación con polvo.

- Se controlará la circulación del personal de la construcción por los lugares delimitados para ello.

- El cierre debe construirse con tabiquería y debe ser hermético, las junturas y holguras deberán sellarse con cinta de embalaje por el interior y exterior. De no ser posible se deberá colocar armazón de madera cubierto por plástico grueso doble y con los mismos sellos de cinta descrito anteriormente.
- Se debe asegurar que las ventanas y puertas se encuentren selladas de modo que no entre polvo a las áreas asistenciales.

- Uso de paños húmedos a la salida de cada sector y bajo las puertas, además éstas siempre deben estar cerradas.

- Durante la realización de trabajos que generen alta concentración de polvo, considerar la utilización de aspiradora industrial con filtro de agua.

- Si es necesario se podrá solicitar instalar un extractor de forma móvil para dejar con presión negativa el recinto e instalación de filtros HEPA.

- Se controlará el ingreso de materiales de construcción y retiro de escombros, los cuales deberán realizarse idealmente en sacos de plásticos cerrados, sin acopio en los pasillos de circulación.

- Para el traslado de escombros, el ascensor no puede ser ocupado en forma concomitante con el traslado de pacientes. Idealmente se debe realizar en horarios inhábiles.

- Se realizará aseo con paños húmedos 3 veces al día en las distintas áreas.

- La unidad de prevención y control de IAAS tendrá la facultad de suspender la obra en caso de que su desarrollo ponga en riesgo a pacientes y /0 no se esté cumpliendo con las medidas recomendadas en esta norma.

Tabla 1: Clasificación de áreas de trabajo

\begin{tabular}{|l|l|}
\hline \multicolumn{1}{|c|}{ Clasificación } & \multicolumn{1}{c|}{ Areas de Trabajo } \\
\hline Bajo Riesgo & $\begin{array}{l}\text { - Areas de oficina no insertas en servicios clínicos } \\
\text { - Espacios públicos } \\
\text { - Otras áreas administrativas }\end{array}$ \\
\hline \multirow{2}{*}{ Riesgo Moderado } & $\begin{array}{l}\text { - Salas de pacientes de servicios generales } \\
\text { - Laboratorios (excepto de microbiología) } \\
\end{array}$ \\
& $\begin{array}{l}\text { - Servicio de alimentación } \\
\text { - Medicina nuclear }\end{array}$ \\
\hline
\end{tabular}


Recomendaciones para la prevención de infecciones relacionadas con demoliciones, - F. Moreno et al

\begin{tabular}{|l|l|}
\hline Riesgo Moderado & - Terapia Física \\
& - Radiología y RM \\
& - Policlínicos \\
\hline \multirow{3}{*}{ Alto Riesgo } & - Cualquier área con pacientes inmunocomprometidos \\
& - Unidades que incluyan pacientes quemados \\
& - Farmacia \\
& - Central de esterilización \\
& - Salas de procedimientos invasivos (hemodinamia, \\
& angiografía, endoscopía, etc) \\
& - Unidades de pacientes críticos (Neonatología, UCIA, UCIP \\
& intermedios) \\
& - Salas de aislamiento \\
& - Oncología \\
& - Pabellones quirúrgicos incluidos, ginecología y oftal \\
& mología \\
& - Preparto, parto y atención de recién nacido \\
& - Emergencia \\
& - Laboratorio Microbiología \\
\hline
\end{tabular}

\section{EVALUACIÓN DE RIESGO}

Se constituirá un equipo multidisciplinario a cargo del diseño inicial de cualquier proyecto de construcción, renovación, remodelación u otras actividades afines (incluye corte de agua programado), que siempre deberá contar con al menos un profesional de unidad IAAS.

\section{SUPERVISIÓN DE LAS MEDIDAS}

La supervisión en la aplicación de estas medidas y su eficacia son responsabilidad de la unidad de prevención y control de IAAS, quienes realizarán actividades como: inspección visual de la presencia de polvo ambiental, verificación del cumplimiento, mantención de las medidas y vigilancia de infecciones asociadas a construcciones. El incumplimiento podrá dar origen a la suspensión de la actividad u obra.

\section{BIBLIOGRAFÍA}

1. MINSAL. Norma para la prevención de infecciones asociadas a modificaciones estructurales y otras actividades que generan polvo ambiental en establecimientos hospitalarios.2011.

2. CDC. Guidelines \& Recommendation for ventilation, construction and renovation of hospitals. 2003.

3. Burghi G, Lemiale V, Bagnulo H, Bódega E, Azoulay $\mathbf{E}$. Aspergilosis pulmonar invasiva en el paciente hematooncológico en las unidades de cuida- dos intensivos: Revisión de la literatura médica. Med Intensiva. 2010; 34(7):459-466.

4. Cruz R, Piontelli E. Enfermedad fúngica invasora en pacientes de cinco hospitales de la Región de Valparaíso, Chile: 2004 a 2009. Rev Chil Infectol. 2011; 28(2):123-129.

5. Iribarren 0, Ferrada 0, Dorn L. ¿Las construciones y obras civiles aumentan el riesgo de infeccion herida operatoria? Rev Chilena de Cirugia: 2004; 3: 210-215. 
Recomendaciones para la prevención de infecciones relacionadas con demoliciones, - F. Moreno et al

6. MINSAL. Revisio'n de evidencia cienti'fica y Londoño G, Alvarez C, editores. Editorial Panamericarecomendaciones para el manejo ambiental de pacien- $\quad$ na. 2010.

tes transplantados de medula osea. 2008.

7. Valderrama S, Ariza B, Osorio J. Medio Am- de las infecciones nosocomiales. WHO/CDS/CSR/EPH/ biente e infección. Infecciones Hospitalarias Malagon- 2002.12. 